\title{
Trayectorias de clases previsibles e imprevisibles. El lugar de la transmisión familiar en la reproducción y el ascenso social en Argentina
}

\author{
Predictable and unpredictable class trajectories. The place of \\ family transmission in reproduction and social advancement in \\ Argentina
}

\section{Leticia Muñiz Terra}

lmunizterra@conicet.gov.ar

Centro Interdisciplinario de Metodología de las Ciencias Sociales. Instituto de Investigaciones en Humanidades y Ciencias Sociales (IdIHCS CONICET). Universidad Nacional de La Plata, Argentina

Recepción: 24 Mayo 2020

Aprobación: 03 Octubre 2020

Publicación: 01 Febrero 2021

Cita sugerida: Muñiz Terra, L. (2021). Trayectorias de clases previsibles e imprevisibles. El lugar de la transmisión familiar en la reproducción y el ascenso social en Argentina. Cuestiones de Sociología, 24, e114. https://doi.org/10.24215/23468904e114
Resumen: El presente artículo busca comprender las desigualdades sociales de clase a través de la mirada dinámica de las trayectorias laborales. La idea central es analizar cómo se configuran las desiguales trayectorias de las clases sociales entendiendo a las mismas como caminos constituidos por un conjunto de decisiones subjetivas que los sujetos van poniendo en juego frente a los constreñimientos externos que se les imponen. En estas transiciones consideramos especialmente el rol de la transmisión familiar en el despliegue de dichas trayectorias. El texto se apoya en una investigación cualitativa para la cual se realizaron 92 entrevistas biográficas a varones y mujeres de distintas clases sociales del Gran La Plata, provincia de Buenos Aires, Argentina. Las historias de vida reconstruidas estuvieron centradas en las trayectorias laborales y educativas de los sujetos entrevistados, trayectorias que nos permitieron recuperar el lugar que la transmisión familiar tiene tanto en las trayectorias de clase previsibles, es decir, en las trayectorias de reproducción social del origen familiar, como en las trayectorias imprevisibles, en este caso las trayectorias de ascenso social.

Palabras clave: Desigualdades Sociales, Clases, Trayectorias.

Abstract: This article seeks to understand the social inequalities of class through the dynamic perspective of labor trajectories. The central idea is to analyze how the unequal trajectories of the social classes are configured, understanding them as paths constituted by a set of subjective decisions in front of the external constraints that are impose. In these transitions we consider especially the role of family transmission in the deployment of these trajectories. The text is based on qualitative research for which 92 biographical interviews were conducted with men and women from different social classes of Gran La Plata, province of Buenos Aires, Argentina. The reconstructed life stories were centered on the work and educational trajectories of the subjects interviewed, trajectories that allowed us to regain the place that family transmission has both in predictable class trajectories, in other words, in the trajectories of social reproduction of the family origin, as in the unpredictable trajectories, in this case the trajectories of social advancement.

Keywords: Social Inequalities, Classes, Trayectories. 


\section{Introducción}

Las investigaciones ${ }^{1}$ realizadas por las ciencias sociales sobre desigualdad, movilidad social y clases tienen una amplia tradición en Argentina. En las dos primeras décadas del siglo XXI, los estudios han enfocado la mirada, en líneas generales, en la movilidad intergeneracional a partir de aproximaciones metodológicas cuantitativas que han abordado el análisis del ascenso y/o reproducción social y de los niveles de apertura o cierre del sistema de estratificación social y su relación con los modelos de acumulación desplegados en la región (Jorrat, 1997, 2000; Kessler y Espinoza, 2003; Dalle, 2010; 2013; Chávez-Molina, Pla y Molina Derteano 2011, Chávez- Molina 2012; Salvia, 2012; Plá, 2016, Benza, 2014).

Los estudios cualitativos sobre clases sociales han recibido, en cambio, una menor atención. Entre ellos sobresalen aquellos que se han dedicado a estudiar los procesos de movilidad descendente de las clases medias durante la década del 90 (Minujin y Kessler, 1995; Kessler y Di Virgilio, 2005), las formas de sobrevivencia de las clases populares tras la pérdida del empleo fabril que han tendido a caer en la pobreza en ese mismo período (Gutiérrez, 2004; Eguía y Ortale, 2007; Salvia y Chávez Molina, 2007; Muñiz Terra, 2012b), la movilidad ascendente entre los mecanismos de cierre que tienden a reproducir la desigualdad en la estructura de clases (Dalle, 2010; Plá, 2016), las inserciones laborales de distintas clases sociales y los sentidos y las fronteras simbólicas de la desigualdad (Gutiérrez y Mansilla, 2015; Assusa, 2019; Jiménez Zunino y Assusa, 2017; Jiménez Zunino, 2019).

Hasta el momento se han desplegado pocos trabajos preocupados por analizar las desigualdades de clase de manera dinámica y se ha evidenciado un desarrollo relativo en relación al lugar de la transmisión intergeneracional en la construcción de las trayectorias laborales de las clases sociales de manera contrastada.

Este artículo se inscribirá en esta perspectiva incorporando, en particular, una mirada diacrónica. Es decir, analizará cómo los sujetos construyen sus trayectorias laborales de clase a partir de un conjunto de decisiones subjetivas que ponen en juego frente a los constreñimientos externos que se les imponen, considerando especialmente el rol de la transmisión familiar en el despliegue de dichos caminos.

A partir de esto, el artículo muestra de qué manera la desigualdad se "hace carne" en las trayectorias laborales de las clases, analizando por qué los distintos grupos despliegan dichos itinerarios y qué lugar ocupa la trasmisión intergeneracional en los caminos ocupacionales desarrollados.

A partir de un estudio cualitativo en que se realizaron 92 entrevistas biográficas a mujeres y varones ${ }^{2}$ de las clases de servicios, intermedias y trabajadoras, siguiendo la estratificación propuesta por Goldthorpe (1992), se visibilizarán las diferentes formas que adopta la transmisión intergeneracional: de los recursos materiales y las posibilidades ocupacionales, de los saberes y oficios, de los valores y aspiraciones, de los esfuerzos y los sueños, y el lugar que ellas tienen en las decisiones tomadas para la construcción de las trayectorias laborales, transmisiones que en algunos casos promueven el desarrollo de trayectorias 
reproductivas y en otros posibilitan desplegar trayectorias no esperadas, que logran superar las pautas de reproducción social que los constreñimientos estructurales y los factores institucionales tienden a construir.

En cuanto al orden de exposición, presentaremos, en primer lugar, el recorte conceptual con el que trabajamos, definiendo de manera concreta las nociones de desigualdades, clases sociales, trayectorias y transmisión familiar. En segundo lugar, desarrollaremos la estrategia metodológica seguida. En tercer lugar, expondremos los resultados de la investigación abocándonos a presentar las trayectorias de reproducción de las clases de servicios, intermedias y trabajadoras, y los itinerarios de ascenso hacia la clase de servicio e intermedia. Finalmente, desarrollaremos las reflexiones finales que reponen los argumentos elaborados y los hallazgos realizados.

\section{1- La teoría de la investigación: perspectivas conceptuales acerca de las desigualdades, las clases sociales, las trayectorias y la transmisión familiar}

En este artículo la desigualdad social es abordada como un fenómeno que se produce como resultado de la articulación/tensión entre distintas escalas: los condicionamientos externos (escala macrosocial), las relaciones y políticas institucionales (escala mesosocial) y las experiencias individuales desarrolladas como resultados de procesos heterogéneos (escala microsocial) (Reygadas, 2004; Dubet, 2011; Therborn 2006; Wilkinson \& Pickett 2009; Savage 2014, Saraví, 2015) que se desenvuelven a lo largo del tiempo y en contextos culturales e históricos específicos.

Consideramos además que las desigualdades son relacionales, ya que necesariamente un colectivo es desigual respecto de otro, con el que suele establecer relaciones de intercambio, dominación o subordinación. En las sociedades capitalistas y de mercado, en las que el trabajo estructura las condiciones de reproducción de la vida y la sociedad en su conjunto, una de las herramientas de análisis más potente para comprender esas desigualdades es el agrupamiento de los individuos en colectivos que ocupan distintas posiciones de la estructura social: las clases sociales y las diferentes formas de estratificación social. Analizar la desigualdad social desde la perspectiva de las clases sociales implica entonces, más allá de la mirada teórica de clases a la que se adscriba (marxista, weberiana, neomarxista, neoweberiana, etc), la delimitación de distintas clases que se relacionan entre sí.

Asimismo, consideramos que las desigualdades sociales se producen $\mathrm{y}$ reproducen de manera multidimensional. Es decir, las desigualdades relacionales de clases están configuradas por distintas dimensiones (trabajo, educación, familia, género, raza, etc.), muchas veces interconectadas entre sí.

De esta forma, desde nuestro punto de vista, las desigualdades se encuentran configuradas por la manera en que las múltiples escalas (macro, meso y microsociales) se articulan y tensionan, por las relaciones de clases particulares que se establecen, y por el entrelazamiento que se produce entre dimensiones (trabajo, educación, familia, etc.). Esa configuración tiene la particularidad, además, de ser dinámica, es decir, de ir transformándose a lo largo del tiempo. 
Esta mirada de las desigualdades sociales, pensadas como procesos interescalares, relacionales y multidimensionales que se producen y reproducen en la temporalidad, puede ser aprehendida a partir de la perspectiva de las trayectorias sociales. Esta aproximación heurística promueve el estudio de los recorridos biográficos de los individuos, que son el resultado de la articulación/ tensión de las distintas dimensiones y escalas (macro, meso y micro) a lo largo del tiempo y el espacio (Muñiz Terra, 2012a).

Así, esta perspectiva de trayectorias sociales de clase supone una mirada subjetiva que considera que es posible comprender la configuración de las desigualdades sociales a partir de recuperar y dar valor a la perspectiva del actor, sus representaciones y cursos de acción. Como ya lo ha dicho Bourdieu (2011), los procesos de socialización y enclasamiento en la sociedad se vinculan con la posición que tienen las familias de los individuos en la estructura social y con la transmisión de patrimonios y recursos económicos, así como también oficios, saberes, oportunidades laborales, modelos de conducta, actitudes, valores, deseos, tabúes, etc. Ese proceso de transmisión ocurre en las familias a lo largo del tiempo de formas más o menos conscientes y más o menos voluntarias, y la recepción y apropiación de aquello que se transmite por parte de los descendientes puede ser también bastante variable (Bertaux y Bertaux Wiame, 1997).

En este artículo centramos entonces nuestro interés en el lugar que ocupa la transmisión familiar (material, social y simbólica) en las trayectorias de clase (laboral) y la forma en que dicha trasmisión es apropiada por los actores sociales. Cuando hablamos de transmisión hablamos de aquello que los miembros de las familias pueden pasar, heredar a las siguientes generaciones (Bertaux y Thompson, 1997), ya sea en términos de capital económico, como cultural, social y simbólico (Bourdieu, 2011). Así, aquello que se transmite, que las generaciones anteriores heredan a sus descendientes lo deseen o no, puede determinar o ser una condición de posibilidad para el despliegue de trayectorias imprevisibles en las generaciones futuras. Esta aproximación desborda y complementa las explicaciones clásicas, que sostienen que las desigualdades sociales y sus posibles reducciones son una consecuencia tanto de la aplicación de políticas educativas inclusivas, o modelos de acumulación redistributivos, como de las movilidades entre el origen y el destino de los individuos, a partir únicamente de la comparación entre la ocupación del padre/madre con la de la persona encuestada. Adoptar esta mirada supone sostener que la reproducción o no de la posición social no es para nada mecánica, sino "un proceso dinámico en el cual los individuos pueden ser entendidos como jugadores que participan del juego de la competencia social generalizada" (Bertaux y Bertaux-Wiame, 1997, p. 64).

Ahora bien, a diferencia de los estudios tradicionales de movilidad social, cuando se piensa la transmisión intergeneracional en el marco de esta perspectiva, no se piensa únicamente en la transmisión de la madre o el padre a los hijos, sino en una trasmisión en el marco de un árbol familiar mucho más amplio, que incluye a los abuelos, tíos, hermanos mayores, etc. La idea, así, es que la transmisión material pero también simbólica (los deseos, las frustraciones, los valores, el prestigio, el reconocimiento, etc.) puede estar encarnada en distintos familiares con los que el individuo tiene más o menos afinidad. Asimismo, resulta significativo señalar que la transmisión simbólica no es un proceso lineal como la herencia material, sino que se va construyendo en la temporalidad, se va 
sedimentando de manera diacrónica (Thompson, 1997). De allí que es posible señalar que la transmisión de parte de un antepasado puede ir acumulándose durante mucho tiempo y en determinados momentos de las trayectorias el individuo decide apropiársela y recurrir a ella. De allí que la transmisión de un abuelo o de un tío puede influir en la trayectoria vocacional o aspiracional de un nieto o sobrino, y promover luego una metamorfosis de la herencia prevista.

La perspectiva de la transmisión familiar se transforma, desde nuestro punto de vista, en una aproximación que propicia una mayor comprensión de la configuración de las desigualdades sociales de clase, y esto aporta herramientas para conocer por qué algunos individuos logran construir trayectorias de ascenso y otros no, escapando o sosteniendo la gama de posibles destinos que les confería su origen familiar de clase.

Las múltiples trasmisiones que recortamos se vinculan con la noción de capitales sociales de Bourdieu (1997, 2011), quien señala la importancia de los capitales económico, social, cultural y simbólico. En particular recuperamos para el análisis las ideas de capital económico (entendida como el conjunto de bienes necesarios para producir riqueza), capital social (relacionado con los recursos reales o potenciales que están unidos a la posesión de una red duradera de relaciones más o menos institucionalizadas de reconocimiento mutuo) y capital simbólico (vinculado con el prestigio, la legitimidad, la autoridad, el reconocimiento, la distinción y la diferenciación). Asimismo, retomamos aquí la idea de Bourdieu (2011, p. 133) referida a que la familia es "uno de los lugares por antonomasia de la acumulación de capital bajo sus diferentes especies y de su transmisión entre las generaciones".

La particularidad del análisis que proponemos está centrada en la idea de que la sedimentación diacrónica de las dimensiones laborales y familiares, es decir, la comprensión de las trayectorias laborales, y del lugar que en ellas ocupa la transmisión familiar y la apropiación por parte de los individuos son dimensiones subjetivas que pueden contribuir junto a las dimensiones macrosociales y mesosociales a aprehender la configuración de las trayectorias de clases, las desigualdades sociales y sus cambios y permanencias en la temporalidad.

Por ello desarrollamos aquí un análisis de las trayectorias laborales de las clases de servicios, intermedias y trabajadoras, centrando la atención en la interpretación de las experiencias subjetivas de los actores sociales, las transmisiones familiares que circularon en sus recorridos y sus correlatos en la construcción de las trayectorias ocupacionales. Ahora bien, dada la extensión posible de este artículo, no desarrollamos con mayor detalle las dimensiones macrosocialles y mesosociales que configuran también las desigualdades. Esto no significa que no las consideraremos, sino que nos concentramos en la dimensión microsocial, recuperando en la argumentación la relevancia que lo macro y lo meso van teniendo en las trayectorias de clase analizadas.

\section{2- Método}

Para este estudio aplicamos una investigación cualitativa centrada en la perspectiva biográfica, en tanto aproximación que posibilita estudiar procesos sociales (las desigualdades sociales) a partir de observar cómo se articulan/ 
tensionan en las historias de vida los constreñimientos estructurales (dimensiones macrosociales y mesosociales) que se imponen a los actores sociales y el mundo de las decisiones, disposiciones, representaciones y acciones que ellos despliegan (dimensión microsocial) a lo largo del tiempo y en el espacio. De este modo, las historias de vida que la perspectiva biográfica permite recuperar son el resultado de múltiples dimensiones o subhistorias (laborales, educativas, religiosas, políticas, de clase, etc.), que se entrecruzan en la temporalidad y que se ven condicionadas y/o propiciadas por elementos estructurales y subjetivos (Muñiz Terra, 2012). En particular reconstruimos y analizamos las trayectorias laborales de las clases de servicios, intermedias y trabajadoras, y el lugar que en ellas ocupa la transmisión familiar intergeneracional.

Para la investigación se realizaron 92 entrevistas biográficas a actores sociales que fueron seleccionados a partir de una muestra intencional basada en los siguientes criterios: que fueran varones y mujeres, de generaciones jóvenes y adultas, que estuvieran ocupados/as y cuyo principal sostén de su hogar fuera de las clases de servicios, intermedias o trabajadoras. El número total de entrevistas a realizar se definió a partir de la saturación de la información construida. El trabajo de campo fue realizado en el aglomerado Gran La Plata, Buenos Aires, Argentina, contexto regional relevante, ya que presenta indicadores laborales similares a los indicadores nacionales y a los de los grandes aglomerados urbanos del país. Esta situación nos permite hacer una extrapolación razonable (Patton, 2002) y hablar de la desigualdad social y de las trayectorias de clase en Argentina. No se pretende así una representación estadística sino una generalización analítica (Znaniecki, 1934)

La asignación de clase fue definida en función de la ocupación del principal sostén del hogar y estuvo orientada por la tradición sociológica vinculada a los estudios relacionales de la estructura y la movilidad social, que históricamente ha operacionalizado a las clases basándose en la ocupación (Goldthorpe, 1987, Erikson y Goldthorpe, 1992).

A continuación, presentamos el esquema que resulta de esta perspectiva mostrando las tres macroclases que distingue y las fracciones de clases que cada una incluye. 
Figura 1

Esquema de Clases

\begin{tabular}{|c|c|}
\hline $\begin{array}{l}\text { I. Clase de servicios nivel superior: profesionales, } \\
\text { administradores y gerentes }\end{array}$ & \multirow{2}{*}{$\begin{array}{l}\text { Clase de } \\
\text { servicios }\end{array}$} \\
\hline $\begin{array}{l}\text { II. Clase de servicios nivel inferior: profesionales, } \\
\text { administradores y gerentes de nivel inferior, técnicos, gerentes } \\
\text { de pequeños establecimientos industriales. }\end{array}$ & \\
\hline $\begin{array}{l}\text { Illa. Empleados no manuales rutinarios de nivel superior } \\
\text { (administración) }\end{array}$ & \multirow{6}{*}{$\begin{array}{l}\text { Clases } \\
\text { intermedias }\end{array}$} \\
\hline $\begin{array}{l}\text { Ilb. Empleados no manuales rutinarios de nivel inferior (ventas y } \\
\text { servicios) }\end{array}$ & \\
\hline IVa. Pequeños propietarios con empleados & \\
\hline IVb. Pequeños propietarios sin empleados & \\
\hline $\begin{array}{l}\text { IVc. Pequeños propietarios y otros trabajadores por cuenta } \\
\text { propia en la producción primaria }\end{array}$ & \\
\hline $\begin{array}{l}\text { V: Técnicos de nivel inferior y supervisores de trabajadores } \\
\text { manuales. }\end{array}$ & \\
\hline VI. Trabajadores manuales calificados & \multirow{3}{*}{$\begin{array}{l}\text { Clase } \\
\text { trabajadora }\end{array}$} \\
\hline VIla: Trabajadores manuales no calificados (no agrícolas) & \\
\hline VIIb. Trabajadores manuales no calificados (agrícolas) & \\
\hline
\end{tabular}

Fuente: Erikson, Goldthorpe y Portocarero (1979).

Ahora bien, en relación a las clases y fracciones de clases de la estratificación escogida, resulta importante señalar que, en contraste con la importancia que estas divisiones tienen para los estudios cuantitativos de movilidad social, para el análisis cualitativo de trayectorias tanta especificidad no resulta significativa, pues no se trata de observar la movilidad de origen y destino entre las fracciones y las clases sociales, sino la configuración de las trayectorias laborales y la forma en que las ocupaciones se van sucediendo en articulación con otras dimensiones (en este texto la familiar), dando lugar a trayectorias de clases distintas. Sin embargo, consideramos interesante considerar a dichas fracciones de clase dado que ellas dan cuenta de distintos segmentos ocupacionales, lo que garantiza una heterogeneidad relevante en el análisis de la composición de las clases.

Por otro lado, una aclaración que resulta importante realizar es que, si bien los/ as entrevistados/as han sido originariamente asignados a una clase en función de la posición en la estructura social del principal sostén del hogar (origen familiar), luego en el análisis de sus trayectorias recuperamos y analizamos sus distintas inserciones ocupacionales a lo largo del tiempo, y, aunque sus trayectorias suelen culminar en inserciones laborales correspondientes a la clase de referencia de 
su familia, no siempre es así, ya que algunos han ascendido. Por otro lado, muchos de ellos/as no desarrollan todo su recorrido ocupacional en trabajos correspondientes a sus clases de origen.

En cuanto al análisis, cabe destacar que desarrollamos un análisis de acontecimientos biográficos y momentos bifurcativos (Muñiz Terra, 2018), en el que, además de la interpretación temática, minuciosa y diacrónica de los relatos recogidos, articulamos esta escala analítica subjetiva con las escalas macrosociales y mesosociales, con la idea de aportar una mirada integral para el estudio de las desigualdades sociales.

El periodo de las trayectorias reconstruido va desde 2003 a 2019, momento histórico que incluye tres gobiernos progresistas (2003-2015) y un gobierno neoliberal (2016-2019). De allí que es posible señalar que las trayectorias analizadas transitaron por un período más largo en esquemas de bienestar proteccionistas, con políticas laborales universalizadoras que tendieron a proteger y a regular el trabajo, y, un período más breve, pero no por ello menos intenso, de un esquema de bienestar liberal que se inclinó por aplicar políticas estatales laborales con una clara tendencia hacia la focalización, la meritocracia y la autogestión individual en el mercado ocupacional.

\section{3- La transmisión familiar en la clase de servicios y hacia ella. Trayectorias laborales en la cúspide de la estructura social.}

En este apartado presentamos la configuración de las trayectorias laborales de la clase de servicios, analizando en particular aquello que las familias legan a sus descendientes, y el lugar que esta herencia tiene en la reproducción social. En particular hacemos una división entre las trayectorias de los trabajadores que provienen de familias de la clase de servicios y las trayectorias de aquellos que llegan a la clase de servicios, es decir, que logran ascender a esta clase social.

\section{1- Trayectorias de inercia reproductiva: el lugar de la transmisión en la clase de servicios}

Las trayectorias laborales en la clase de servicios, que realizan quienes provienen de familias que ocupan esa posición en la estructura social, se inician habitualmente luego de los 20 años de edad y se despliegan en empleos temporales y con horarios reducidos en los que los jóvenes se insertan con la voluntad de obtener ingresos para sus gastos personales mientras van a la universidad.

Los estudios universitarios forman parte de una larga transición escolar que las familias planifican para sus hijos/as. Desde el momento de la elección de las escuelas secundarias hay una preocupación por la orientación que tomará la formación de los niños y hay tradiciones familiares que intentan reproducirse a la hora de escoger las escuelas a la que irán.

Pero se sabía que el nivel de la escuela pública ya era bastante malo, entonces o vos entrabas a una universitaria, para tener una buena formación, o te ibas a un colegio privado. Como que así era el mandato familiar, por lo menos (Entrevista $n^{\circ} 54$. Reproducción de la clase de Servicios.) 
Las trayectorias educativas de estas clases suelen caracterizarse por ser continuas, es decir, que presentan linealidad en términos educativos, en tanto se expresan a partir de transiciones pautadas y sincronizadas, siguiendo las expectativas y los tiempos institucionalizados por las políticas educativas del país. El paso por la secundaria es vivido y transmitido a nivel familiar como una etapa más, que debe conducir a la universidad y a un título profesional.

En mi casa, no había ninguna posibilidad de que alguien no terminara el secundario, así que medio que era lo que había que hacer para ir a la universidad (Entrevista $n^{\circ}$ 55. Reproducción de la clase de servicios)

El conocimiento de idiomas, que luego resulta una posibilidad para trabajar o ganar algún recurso extra, también es una de las oportunidades que las familias se ocupan de legar a sus hijos

cuando estaba haciendo la carrera juntaba, por ahí, algún "mango", haciendo algún trabajito de traducción, como yo... como mi vieja es profesora de inglés y me mandó a estudiar de muy chiquito y manejo el idioma... además me gustan los idiomas, así que... me daba maña con eso de la traducción (Entrevista $n^{\circ} 70$. Reproducción de la clase de servicios)

Las familias suelen también brindar distintos apoyos económicos que colaboran en la preparación para el futuro trabajo o en el desarrollo de las trayectorias laborales. Las mensualidades, la compra de vehículos para facilitar el traslado por la ciudad, el pago de viajes de estudio o trabajo en el exterior, etc. aparecen así de manera recurrente en sus relatos.

\begin{abstract}
Mi vieja me apoyaba. Entonces, si yo me tenía que comprar un instrumento, ella me lo compraba, o la cámara filmadora me la compró ella... entonces, si bien no había un apoyo de la boca para afuera, sí había un apoyo concreto material que nunca faltó. Si yo para ir a laburar necesitaba el auto, me lo prestaba, iba a la escuela si no se me hacía muy tarde... Después, cuando fuimos más grandes, mi vieja y mi viejo, a mi hermano y a mí, nos compraron un vehículo, una camionetita, entonces ya disponíamos, íbamos para acá, para allá, entonces se hacía mucho más fácil ir a trabajar (Entrevista $n^{\circ} 54$. Reproducción de la clase de servicios)
\end{abstract}

Asimismo, entre las familias de las clases de servicios se transmiten ciertos saberes o vocaciones que pueden ser luego puestos en juego el mercado de trabajo. En algunos casos los hijos estudian lo mismo que sus padres/madres o tíos, o se orientan por profesiones asociadas. Las carreras de ingeniería, derecho y medicina son las más habitualmente reproducidas.

Sí, casi toda mi familia son abogados. De hecho, yo soy el único de mi familia más cercana, entre primos y hermanos, que no tiene un título universitario, después todos tienen título universitario, y son, la mayoría, abogados o médicos (Entrevista $n^{\circ} 72$. Reproducción de la clase de servicios).

Luego de finalizar los estudios universitarios, las trayectorias laborales presentan distintos estadios, siempre vinculados a momentos de ocupación que, aunque inicialmente no tienen una larga duración (dos o tres años), luego tienden a estabilizarse, produciendo cambios en las inserciones ocupacionales que representan mejoras en las condiciones de trabajo y en los salarios o retribuciones alcanzadas.

En ese camino, un legado que también aparece de manera recurrente es la oportunidad de ingresar a trabajar en el Estado aportada por algún familiar. La 
relevancia de la clase de servicios en el empleo público en la ciudad de La Plata, que ha sido ya mencionada en otros trabajos (Muñiz Terra, Pla y Iucci, 2019), aparece de manera recurrente en algunos relatos, que valoran esta posibilidad como algo dado y transmitido de generación en generación.

Mi vieja trabaja dentro de esta estructura del Estado, pero yo tengo mi familia que también está trabajando en Poder Judicial, en otra estructura, que depende de la Corte y no de lo que es la Procuración. Entonces, sí, no solo mi vieja está laburando acá, sino que vos decís Moreno y van a decirte “ah, mira, bla, bla”. Acá se dice que uno tiene padrinos.... hay una gran mayoría que tiene esta cuestión de influencias. (...) en similitud con mi viejo, que la trayectoria de él es medio familiar, y yo también ingreso en el Poder Judicial porque tengo contactos familiares. Si bien están las condiciones dadas de la parte de la idoneidad y de cierta cuestión oficial (todos abogados que trabajan en el Poder Judicial), si yo no hubiese tenido el contacto familiar, no sé cómo hubiera ingresado (Entrevista $n^{\circ} 54$. Reproducción de la clase de servicios)

Con el paso del tiempo las inserciones laborales van adquiriendo cierta formalidad y se van transformando en relativamente homogéneas, hasta alcanzar, en líneas generales, cargos jerárquicos en sus puestos de trabajo, asociados a la vez a sus carreras profesionales y con buenas condiciones de contratación, con estabilidad y seguridad social.

En suma, en las trayectorias de las clases de servicios la transmisión se juega en las oportunidades que las familias les dan a sus descendientes y que son aprehendidas por estos para insertarse y/o desplegar trayectorias laborales en la misma clase social que sus progenitores. Lo que se transmite, en líneas generales, tiene que ver con un conjunto de oportunidades formativas, materiales y laborales que posibilitan cierta inercia reproductiva al permitir a la clase de servicios reproducir su posición de clase en la estructura social.

\subsection{Trayectorias aspiracionales: el lugar de la transmisión en el ascenso social hacia} la clase de servicios

Las trayectorias laborales hacia las clases de servicios que realizan quienes provienen de familias de clases intermedias o trabajadoras habitualmente comienzan en la adolescencia, cuando se insertan en trabajos informales y temporarios mientras van a la escuela secundaria. Estas primeras inserciones se realizan habitualmente en el sector servicios (comercios) y son motivadas por el deseo de adquirir recursos para gastos propios que las familias no pueden solventar.

trabajaba con libritos donde se venden los productos, con una marca, que creo que no está más, que se llamaba René Briam. Después seguí con Avon, dos años después. A los quince arranqué con eso. (...) Me llevó a arrancar que yo vengo de una familia humilde, que tengo siete hermanos más, mis papás laburantes, los dos, pero ellos por ahí no podían darme cosas que quizás yo quería, como una ropa, esto o lo otro (Entrevista $\mathrm{n}^{\circ} 50$. Ascenso hacia la clase de servicios).

El tránsito por los estudios superiores forma parte de una de las transmisiones más recurrentes de los familiares del grupo que asciende a la clase de servicios, quienes valoran especialmente la formación universitaria y explican parte de sus dificultades laborales por el hecho de no haber podido realizar este tipo de formación. Dicha experiencia y valoración los lleva a transmitir a sus hijos la 
importancia de alcanzar la titulación de grado como llave para la realización de una buena carrera ocupacional. Estas ideas son acompañadas, en algunos casos, por las transmisiones institucionales, es decir, por la transmisión de los docentes de las escuelas secundarias de la relevancia de ir a la universidad.

yo quería ser universitaria, por muchas cosas... En mi casa siempre me inculcaron que tenía que tener una carrera, el colegio se encargó todos los días de inculcarnos que teníamos que tener una carrera universitaria, y además porque yo quería ser universitaria, porque quería pertenecer a ese grupo que yo no era. Quería ascender de clase social y sabía que la única forma, ya de chica lo sabía, era estudiando (Entrevista $\mathrm{n}^{\circ} 6$. Ascenso desde clase intermedia a clase de servicios)

Las trayectorias educativas de este grupo de la clase de servicios son lineales hasta que llegan a la universidad, momento en que dicha progresión se ve en varios casos interrumpida o detenida por la imposibilidad de compatibilizar trabajo y estudio. Sin embargo, algunos entrevistados logran culminar sus estudios superiores con mucho esfuerzo y un poco de retraso.

entre el trabajo y la carrera no podía. Porque entraba a trabajar a las ocho de la mañana y volvía a las diez de la noche, o sea que volvía a mi casa a estudiar, dormía dos o tres horas, había bajado mucho de peso, no daba más, tenía que optar. Y, sinceramente, no me podía bancar sola, mi familia no me podía bancar el estudio si no trabajaba (Entrevista ${ }^{\circ} 50$. Ascenso desde la clase trabajadora a la clase de servicios).

La UCA no tenía buena referencia, no era grande, sabía que era más fácil, pero no me gustaba la idea de quedarme con hacer algo pobre, yo quería hacer algo grande para mí, tampoco es que me costara tanto, pero quería hacer algo para mí... por eso me quedé en la Universidad de La Plata, que era pública y tenía prestigio. Y me recibí de licenciada en administración de empresas luego de varios años... (Entrevista $n^{\circ} 6$. Ascenso desde clase intermedia a clase de servicios)

Las trayectorias laborales que llegan a construir estos trabajadores a lo largo del tiempo se caracterizan por ser formales y suelen desarrollarse de manera independiente en el ámbito privado. El trabajo en el sector público no parece una opción a pesar de que algunos de sus progenitores eran trabajadores estatales.

busqué otro tipo de adrenalina, otro tipo de riesgos... Desde la facultad hasta el trabajo, en todo busqué cosas con más riesgos porque tuve oportunidades de entrar al Ministerio donde laburaban mis viejos, pero no quise nunca. Siempre, de asumir más riesgos, más adrenalina (Entrevista $\mathrm{n}^{\circ} 25$. Ascenso desde clase intermedia a clase de servicios).

La idea de buscar un empleo distinto al familiar o la imposibilidad de recibir un empleo público hacen que sus trayectorias se canalicen al empleo en el sector privado, donde adquirieren un conjunto de saberes vinculados con las dinámicas empresariales, que luego utilizan en sus emprendimientos propios.

Aparte, había trabajado también haciendo supervisión de proyectos de inversión... Había trabajado en... no me acuerdo, otra cosa... para Conicet. Y eso sumó, en lo que hacía yo ya tenía clientes, entonces sumó mucho eso, que yo estaba trabajando en la ventanilla de una empresa, entonces yo era la encargada de la ventanilla de la recepción de proyectos de empresas. Entonces, como se dieron cuenta que yo tenía capacidad para convocar empresas, supongo que eso ha hecho que me llamen del otro trabajo. Y así fui aprendiendo un montón de todos los lugares en los que estuve (Entrevista $n^{\circ}$ 6. Ascenso desde clase intermedia a clase de servicios). 
Algunas desavenencias con los jefes de las firmas en las que se desempeñaban o la voluntad de tener sus propios emprendimientos los llevaron, en líneas generales, a decidir iniciar un camino propio inaugurando sus primeras empresas. Para el desarrollo de estos emprendimientos pusieron en práctica tanto los aprendizajes realizados en sus trabajos previos como las experiencias familiares en el desarrollo de negocios.

tuve algunas cositas que me marcaron bastante, que cuando fui más grande lo fui viendo. Una fue la apertura comercial que me dio desde que... mi papá hubo un tiempo que dejó la obra y abrió una verdulería en casa. Y, aparte de la secundaria, yo trabajaba ahí. Esa verdulería se transformó en un almacén y después en un mercadito, tipo supermercadito, y había una especie de secuencia, primero de responsabilidades, y después de secuencia de apertura, limpieza, carga de mercadería. Pero después nos fundimos... porque no sabíamos manejarlo...pero bueno, aprendí que había que tener un orden desde muy joven y también había que tener responsabilidad (Entrevista ${ }^{\circ} 46$. Ascenso desde la clase trabajadora a la clase de servicios).

La frustración familiar se transforma así en una transmisión de la importancia de la responsabilidad y de aquello que hay que hacer mejor para que pueda funcionar.

mi papá; tengo mucho de él, que es el emprendimiento, y se lo agradezco. O sea, soy como su hilo, lo que deja en la tierra soy yo con esto, y está bueno porque siempre voy por más, me gusta tener más cosas. Pero, a su vez, por características propias o porque he estudiado y me he formado en negocios, algo que mi papá no tenía, y porque siempre fui más tradicionalista... No sé si esa es la palabra, pero siempre fui más precavida, nunca fui de gastar más de lo que tengo (Entrevista $\mathrm{n}^{\circ}$ 6. Ascenso desde clase intermedia a clase de servicios).

Una de las ideas recurrentes que aparecen en los relatos de los trabajadores que ascendieron a la clase de servicios es la necesidad de ir por "más" que les fue transmitida de manera explícita por sus familiares. Los deseos de una mejor situación laboral para sus descendientes se transforman en estas clases en un legado explícito que circula en torno a la idea de siempre "buscar más", y ser el "mejor en lo que se hace", como claves para alcanzar buenas condiciones de vida.

Ahora bien, para que estas transmisiones tengan sentido, es necesario que los destinatarios puedan apropiarse del mandato y logren alcanzarlo. De lo contrario, dicha transmisión pierde consistencia o puede conducir a la frustración de la generación receptora.

En las trayectorias hacia la clase de servicios reconstruidas, dicha transmisión tuvo un rol relevante, en tanto fue incorporada como una forma de ser y estar en el trabajo y fue reivindicada como un legado familiar positivo.

hay un montón de otras cosas que las vivo cotidianamente y a mí me ayudan como para decir "vamos por más" (...) A mí siempre mi papá me dice "que mejor que decir, hacer. Y mejor mostrar tu producto, para después pedir sobre tu producto” ... Y es lo que tratamos de hacer (...) Y de ahí, yo creo que el espíritu de querer hacer cosas y de querer progresar es la similitud que yo encuentro con mi papá. Él es un continuo luchador y de querer generar cosas. Yo me considero en eso muy parecido (Entrevista $\mathrm{n}^{\circ}$ 46. Ascenso desde la clase trabajadora a la clase de servicios).

En estas trayectorias la movilidad ascendente respecto de la clase de los padres es explicada en líneas generales como resultado de las transmisiones familiares recibidas, sus méritos, los esfuerzos y talentos personales sin hacer mención 
a la existencia de una serie de institucionales sociales extrafamiliares como soportes. Si bien la educación pública en todos los niveles, pero sobre todo en la universidad, y un contexto laboral o empresarial favorable han sido una condición de posibilidad para el despliegue de sus trayectorias laborales, estas no aparecen reconocidas en los relatos. A pesar de que el mayor crecimiento laboral se produce en estas trayectorias en los 2000, momento de crecimiento económico y productivo del país (Varesi, 2009), el encadenamiento de oportunidades para el ascenso/éxito no fue identificado como una ventaja estructural y contextual por las personas entrevistadas, sino como producto del esfuerzo personal, las capacidades propias y adquiridas, la transmisión familiar y la voluntad de crecer y superarse.

En estas trayectorias de la clase de servicios se evidencia, en síntesis, que la transmisión es más simbólica que material, vinculada a deseos, vocaciones, aspiraciones, proyecciones y moralidades familiares, que son apropiadas por los entrevistados y que ocupan un lugar importante, en tanto orientan en distintos momentos las decisiones y direcciones que van tomando estos recorridos de ascenso, colaborando así en el cambio del destino socialmente esperado de estas trayectorias de acuerdo a su origen social.

\section{4.- La transmisión en la clase intermedia y hacia ella. Trayectorias laborales entre la cúspide y la base de la estructura social}

En este apartado presentamos la configuración de las trayectorias laborales de la clase intermedia, con la mirada enfocada en la transmisión familiar y en la importancia que esta adquiere en la reproducción y el ascenso social. Desarrollamos, en primer lugar, las trayectorias de los trabajadores que provienen de familias de la clase intermedia, y, en segundo lugar, las trayectorias de aquellos que provienen de la clase trabajadora y llegan a la clase intermedia.

\subsection{Trayectorias de inercia reproductiva: el lugar de la trasmisión en la clase intermedia}

Las trayectorias de la clase intermedia se presentan de manera heterogénea, pues algunos realizan trabajos manuales $\mathrm{e}$ industriales y otros tienen ocupaciones asociadas a la administración y los servicios.

Para ambos grupos la trayectoria educativa incluyó la culminación de la escuela secundaria y la incursión en estudios universitarios y terciarios. La obtención del título secundario implicó una superación del nivel educativo alcanzado por los padres, que se estimulaba y transmitía desde el hogar familiar.

En esa época, yo pensaba que no servía para nada el colegio, pero porque en esa época uno está en otra cosa, cuando está en la adolescencia como que uno tiene otra visión de las cosas, y yo la escuchaba a mi mamá diciendo que lo más importante y la única obligación que teníamos era estudiar y que era una posibilidad que no todos tenían (Entrevista $n^{\circ} 41$. Reproducción de la clase intermedia).

El ingreso a la universidad fue realizado con sacrificio y combinado con la necesidad de trabajar. En varios casos, ante las necesidades familiares de recursos 
económicos, el ingreso al mercado laboral se había producido durante la escuela secundaria.

Sí, sí, trabajaba... de niñera. Casi me quedo libre, tercero o cuarto, re faltaba a la escuela, mal, trabajaba y estaba cansada. (...) Yo salía del colegio, ponele, y comía, qué se yo... estudiaría, haría las tareas y me iba a trabajar (...) en casa no había un mango, básicamente. Siempre costaba, todo costaba... Mi vieja se quejaba mucho de la plata, que esto, que lo otro, no podíamos acceder a tener zapatillas (solloza), entonces trabajar, qué se yo... (Entrevista ${ }^{\circ} 30$. Reproducción de la clase intermedia).

La temprana inserción ocupacional de estos grupos significó el advenimiento de dificultades para realizar una trayectoria educativa universitaria "ideal" o teórica (Teriggi, 2015), es decir, para tener un desempeño continuo; incluso en muchos casos supusieron su abandono.

Para el ingreso y la permanencia en los estudios superiores los/as entrevistados/ as realizaron inicialmente elecciones disciplinares vinculadas a los mandatos o deseos familiares, o escogieron sin un conocimiento concreto, pero luego se produjeron cambios para ajustar las expectativas a los proyectos educativos. Aunque el apoyo económico de padres y madres para la continuidad educativa fue en este grupo limitado, en algunos casos otros vínculos intergeneracionales acudieron en auxilio como soporte.

Cuando terminé el secundario, empecé a estudiar maestra jardinera, me fui de la casa de mis viejos, y la facultad me la pagaba mi abuela. Ahí en la facultad, que era un instituto privado. E: ¿ ¿nn instituto privado para estudiar...? N: Maestra de nivel inicial. Cuando empecé ahí, como me lo pagaba mi abuela, yo no recibía ninguna ayuda de mis viejos (Entrevista $n^{\circ} 60$. Reproducción de la clase intermedia).

En cuanto a las trayectorias laborales de esta clase, resulta significativo señalar la importante diversidad que presentan, pues se desarrollan tanto en puestos administrativos públicos o privados, que comienzan siendo precarios y llegan con el tiempo a ser formales, como por cuenta propia, con formas precarias de contratación pero que permiten con los años alcanzar cierta estabilidad y acceso a la seguridad social, aunque no a una carrera laboral ascendente.

Las redes familiares y de conocidos constituyen un capital social que funciona como soporte para las búsquedas laborales y la contratación, a través de las recomendaciones. Así, si bien poseían ciertas credenciales educativas, como el título secundario (bachiller o técnico), o en algunos casos el nivel universitario incompleto, estas no se conjugaron necesariamente como ventajas a la hora de obtener mejores puestos de trabajo; prima, en cambio, la oportunidad trasmitida por algún familiar.

yo creo que el futuro mío, sabía desde chico que iba a terminar trabajando acá, porque toda mi familia trabajó acá. Mis viejos se conocieron acá, mis abuelos trabajaron acá, o sea, que digamos mal o pronto decirlo, esta empresa desde siempre. Así que por eso yo estaba seguro de que en algún momento iba a entrar acá (Entrevista $n^{\circ} 92$. Reproducción de la clase intermedia).

De esta forma, un legado que aparece de manera recurrente en esta clase social es la oportunidad de ingresar a trabajar en un ministerio o dependencia pública o en empresas privadas en las que trabajaban sus familiares.

En las trayectorias de la clase intermedia, la transmisión se juega, en síntesis, en las oportunidades educativas y laborales que las familias les dan a sus 
descendientes y que son aprehendidas por estos para insertarse y/o desplegar trayectorias laborales en la clase social de origen. Al igual que en la clase de servicio, estas oportunidades dan lugar a cierta inercia reproductiva, al posibilitar que la clase intermedia reproduzca su posición de clase en la estructura social.

\section{2- Trayectorias ambivalentes: entre las herencias y la resignificación del legado en el ascenso hacia la clase media}

Las trayectorias laborales de quienes ascienden desde la clase trabajadora a la clase media son también muy heterogéneas y suelen iniciarse en la adolescencia, mientras concurren a la escuela media.

Mientras las transiciones por los estudios secundarios suelen ser desplegadas por todos los entrevistados de manera continua, el pasaje por el nivel superior es solo realizado por algunos, aunque en forma intermitente o con interrupciones prolongadas. Las transmisiones familiares en términos educativos de padres y madres, pero también de otros miembros de la familia como los tíos y los abuelos, se pusieron en juego en este grupo a través del estímulo para que alcanzaran la terminalidad educativa media, y, en algunos casos, para que iniciaran los estudios superiores.

a mí en mi familia me estimularon mucho por ese lado, cuando era chico me
regalaban libros y si lo terminaba me regalaban otro, entones tenía una biblioteca de
libros, y decía: "bueno ya terminé este"; me traían otro, (...) seguía leyendo y ya como
que después de que adquirís eso ya te queda viste, es importante eso. De hecho, mi
viejo, la familia de mi viejo era pobre, pero era muy culta (Entrevista $n^{\circ} 39$. Ascenso
desde clase trabajadora a la clase intermedia).
Un tío que siempre me pareció muy interesante, que no vive en La Plata, quedó
despedido con un montón de gente cuando cerraron fábricas. En la familia lo
cargaban mucho porque era peronista, había seguido a Menem incluso... (...) Me
contaba mucha historia, al loco le gustaba mucho informarse, leer. Y, aparte, me
contaba mucho de historia sindical; él había sido delegado de fábrica. Y bueno, me
re copaba historia de chica, entonces en un momento era como muy claro que iba a
seguir historia (Entrevista no $n^{\circ}$ 1. Ascenso desde clase trabajadora a la clase
intermedia)

En cuanto a las trayectorias laborales de esta clase social, cabe señalar que son diversas, pues se observa la configuración de distintos caminos ocupacionales. Los primeros trabajos fueron conseguidos en la adolescencia, con el objetivo de poder solventar los gastos personales o para ayudar en la economía familiar. Estas inserciones se desarrollaron siempre en el sector informal e implicaron la realización de diversas labores: atención al público, colaboración en talleres mecánicos o de artesanías, ventas, etc. Como estas ocupaciones se producían en forma paralela a los estudios secundarios, los entrevistados debían atravesar una jornada diaria intensa que se dividía entre el mundo laboral y educativo.

Esta situación se prolongó en el tiempo hasta que iniciaron los estudios superiores, momento en que tomaron la decisión de dejar de estudiar, dado el estrés y el cansancio producido por esta división y los escasos rendimientos académicos alcanzados.

Luego de las primeras incursiones ocupacionales, y con mayor tiempo disponible por el abandono de los estudios superiores, las inserciones laborales se fueron encaminando hacia cierta estabilidad y formalidad en las condiciones laborales. 
Los saberes utilizados para el trabajo fueron adquiridos en las escuelas técnicas a las que acudieron, o de manera informal, a través de la observación y de la práctica de los oficios familiares o en las ocupaciones alcanzadas, que luego pudieron poner en juego, en líneas generales, cuando decidieron insertarse como cuentapropistas. La herencia familiar se pone en juego aquí en el estímulo a la terminalidad educativa en escuelas técnicas y en los saberes que se aprenden en el hogar y se resignifican a lo largo de la trayectoria laboral ascendente.

\begin{abstract}
Mi mamá, cuando vivía, ella también trabajaba en la máquina, lavaba ropa ajena, era ama de casa, hacía comida, muchas cosas para ayudarlo a mi papá, que siempre fue trabajador de curtiembre. Creo que la costura viene de herencia, la máquina que teníamos en casa era de mi mamá (...) La máquina de coser la agarraba cuando estaban durmiendo, porque allá se duerme la siesta, a escondiditas iba y pedaleaba, me tenía loca la máquina, pero no nos dejaban. (...) Ahora en mi trabajo aplico mucho eso que aprendí en casa, a escondidas, mirando (Entrevista $n^{\circ} 14$. Ascenso desde clase trabajadora a la clase intermedia).
\end{abstract}

En estas trayectorias la inserción como cuentapropistas se transforma en una oportunidad diferencial que les ha permitido alcanzar buenas condiciones laborales. Ante la imposibilidad de acceder a un trabajo a través de la herencia familiar, recurren a las redes sociales más cercanas para construir recorridos distintos a los de sus progenitores.

Por otra parte, el legado filial se materializa en estas trayectorias a contraluz, es decir, como un espejo que muestra aquello que no se desea y que ayuda a distanciarse de la posición familiar en la estructura social.

Los dos (el padre y la madre) necesitaron una estructura para hacer sus trabajos, y yo creo que la fui buscando en la gente que iba conociendo y en las cosas que hacía, como armar una mía (...) formas de hacer las cosas o encararlas. Incluso en mi familia, no veo a alguien más que pueda hacer algo como me metí a hacer yo por las mías, tengo esa mirada. En las reuniones familiares como que les cuesta, les comparto, pero siempre es así... (Entrevista ${ }^{\circ} 3$. Ascenso desde clase trabajadora a la clase intermedia).

Veo a mi papá con cierta nostalgia, en el final de su época tuvo que trabajar de sereno, cuidando una fábrica. No fue acumulativo su experiencia, no le sirvió de nada. Llegó un momento en el que se le acabó el trabajo tan bueno que tenía, y tuvo que trabajar de lo que fuera. En mi caso, yo lo veo acumulativo. Es como una carrera esto de joyería, de dar clases, lo veo como acumulativo... (Entrevista $n^{\circ} 17$. Ascenso desde clase trabajadora a la clase intermedia).

El rechazo de lo legado se traduce aquí en un distanciamiento de la transmisión familiar que promueve una resignificación de aquello que se percibe como una herencia no deseada, que hay que recuperar, pero para transformarla y reconducirla en una dirección distinta. En este contexto, el trabajo como sacrificio, inherente a la cultura laboral de la clase trabajadora, es asimismo cuestionado por quienes ascienden a la clase intermedia, que ven el trabajo como una posibilidad de cierta realización personal.

Y con mi viejo fue muy raro porque él es de la idea del trabajo como sacrificio, y a mí me costó un montón despegarme de eso, terapia de por medio. Eso como que el laburo te tiene que doler viste para que valga. Y ahora, recién ahora, estoy en otro momento, pero he estado así muy emparentada con esa idea de trabajo exagerada, ¿viste? (...) Y bueno, será que hay que trabajar más, será como que..., ¿me entendés? Como que, ¡cuánto más querés que trabaje? ¡No puedo más (...) Y bueno es así... y bueno habrá que esforzarse más... y bueno habrá que trabajar más horas... y bueno.... 
No, yo no quiero eso para mi vida, pasármela laburando, veo el trabajo como algo más que me permite desarrollarme (Entrevista $n^{\circ} 21$. Ascenso desde clase trabajadora a la clase intermedia).

Una cuestión que aparece también en las valoraciones de quienes ascienden a la clase intermedia es la tendencia a subrayar las capacidades personales puestas en juego para desplegar su camino laboral, poniendo en un segundo plano los soportes institucionales con que contaron. La educación recibida es reconocida como una herramienta importante, pero a la que es necesario aportarle un sentido apropiado, acorde a la trayectoria laboral de ascenso social que se espera construir. Las posibilidades e imposibilidades atravesadas han sido así interpretadas como consecuencias de sus acciones personales y de la resignificación educativa transitada, sin considerar los contextos mesosociales y macrosociales que les acompañaron.

yo considero que amplié mi visión y fue por esa inquietud de siempre conocer más y de insistir y de ser perseverante con las ideas que se me van ocurriendo y, sinceramente, logré lo que hasta ahora vengo haciendo de esa manera, por convicción de lo que me gusta hacer y con grandes valores, que bueno fueron parte de la educación, desde ya, de eso estoy totalmente agradecida (...). Seguramente estoy cagando algo del paradigma familiar (ríe), pero lo es, entonces yo cuando voy veo una realidad familiar que es distinta a mi realidad, que no la critico pero es bastante cómoda ¿verdad?, es como quieta, no hay mucho cambio, no hay movilidad, y yo acá es como que todo el tiempo he estado como súper estimulada, pero porque ha sido siempre una inquietud mía moverme, hacer, buscar, conocer, sociabilizar, me encanta" (Entrevista ${ }^{\circ} 42$. Ascenso desde clase trabajadora a la clase intermedia).

En estas trayectorias hacia la clase intermedia se observa, en suma, que la transmisión familiar adquiere distintas aristas, pues, mientras por un lado el legado se centra en las oportunidades educativas de acceso y terminalidad del nivel medio, y en algunos saberes que pudieron ser trasmitidos, por otro lado, se advierte un distanciamiento de la herencia vinculada a los valores de la clase trabajadora, que es cuestionada y resignificada en el camino de ascenso y diferenciación de la posición familiar de origen.

\section{Las trayectorias de la clase trabajadora. El lugar de la transmisión en la base de la estructura social}

Analizamos aquí las trayectorias laborales de aquellos que provienen de familias de clase trabajadora y desarrollan también su recorrido ocupacional en el marco de esta clase.

Las trayectorias de los trabajadores, que se ubican en la base de la estructura social, suelen presentar pocas credenciales educativas; en algunos casos primario incompleto, y en otros secundario incompleto. Sus trayectorias laborales, que son muy extensas, se inician durante la infancia (antes de los 12 años de edad).

Los niños dejan la escuela ya sea por la falta de recursos por parte de las familias para acompañar la escolaridad o por la necesidad de que comiencen a trabajar.

Y mi tía, la que nos crio, a veces no tenía y lo que mandaban de acá era para comer, y salíamos a trabajar y no había para comprar lo que te piden en el colegio, y por ahí por ese motivo me salí. Porque ibas y dabas la misma excusa y a veces los maestros... unos te entendían y otros no. Así que... por ese motivo..." (Entrevista n ${ }^{\circ} 16$. Reproducción de la clase trabajadora). 
Porque ya no... mi papá y mi mamá ya no me apoyaban. Hasta octavo fui y listo. Me decían "si vos querés estudiar, estudiá sola". Me decían, "por lo menos ustedes ya saben algo para defenderse”. A mi mamá no la dejaron estudiar, y para nosotros... estaba bien que ella nos ha hecho estudiar, leer, todas esas cosas para defenderse (...) me salí y empecé a trabajar" (Entrevista $n^{\circ} 19$. Reproducción de la clase trabajadora).

Las inserciones laborales tempranas suelen ser breves y con una alta rotación por muchos y diversos empleos, todos ellos precarios e informales, asalariados o por cuenta propia, y en general manuales o asociados al trabajo doméstico.

muchos hermanos tengo. Nosotros somos doce, ya quedamos menos... Así fue que estuve hasta los once años con mi mamá, y esta hermana que me trajo a Argentina se fue allá al campo, junto a mí, y me dijo que ya era grande para estar ahí (...) Y me dijo "te voy a llevar a Asunción. Vas a trabajar”. Fue así que a los once se fue a llevarme del campo (...) Me vine a Asunción, trabajé de niñera porque otra cosa no sabía hacer, porque tenía once años... Después de niñera, hice de limpieza en Asunción. Muchos años trabajé de empleada doméstica ahí, limpiar, cocinar... (Entrevista ${ }^{\circ}$ 28. Reproducción de la clase trabajadora).

En algunos casos los saberes necesarios para la ejecución de las tareas laborales fueron adquiridos de manera empírica e informal, a través de la observación y de la práctica y error. En otros casos, dichos conocimientos laborales formaban parte de la herencia familiar, de aquello que padres y madres trasmitían, a veces sin darse cuenta, en la vida cotidiana.

Aprendí de la gastronomía y de las cosas con ellos. No es que mi familia me enseñó manualidades, suvenires, tejer. Aprendí de verlos a ellos: se han caído, levantado, caído, levantado... por los diferentes gobiernos. Es eso. De alguien lo aprendí y ahora se los enseño a mis hijos... (Entrevista $n^{\circ} 44$. Reproducción de la clase trabajadora).

Las oportunidades laborales circulan también es esta clase como una herencia que se transmite de generación en generación. Oportunidades vinculadas a los trabajos precarios e informales que desarrolla la clase trabajadora.

siempre me interesó porque toda mi familia laburó allá...este...mis dos abuelos, mi tío o sea el hermano de mi papá y mi tía, la hermana de mi mamá, laburaron allá, bah y.... siempre me generó intriga lo que era ahí "adentro" desde que era chiquito. Y bueno, mi viejo me vino con la respuesta esa de que iba a trabajar ahí (...) La intriga que tenía de chico de saber qué era ahí adentro y bueno, me llevó también todo lo que es mi familia que también mis abuelos laburaron allá (Entrevista $n^{\circ} 76$. Reproducción de la clase trabajadora).

Sin embargo, algunos trabajadores, conscientes de las malas condiciones laborales que viven, proyectan y trasmiten a sus hijos el deseo de alcanzar una mejor situación ocupacional, y que dejen el trabajo manual y a la intemperie porque es muy desgastante y agotador.

Yo quiero que sean mejor que yo. Que no sufran tanto en la quinta. Por eso le exijo mucho a Juana el estudio. Que sea algo en la vida y también, sí, la llevo a trabajar, para que sepa que cuesta la plata, que no está para agarrar y tirar (Entrevista $n^{\circ} 16$. Reproducción de la clase trabajadora).

En general, la posición de las personas de clase trabajadora que entrevistamos supone mejores condiciones de vida respecto de su familia de origen, que no tuvo acceso a la educación formal y en algunos casos tiene un origen rural muy pobre. Sin embargo, esto no implica que logren ocupaciones laborales formales y seguras, 
sino que se insertan en un cuentapropismo informal que en ocasiones despliegan en la búsqueda de mitigar las condiciones de explotación que sufren cuando son empleados de manera precaria.

En algunos casos el cuentapropismo informal es además una estrategia para garantizar, por un lado, la continuidad laboral, y, por otro lado, para conciliar trabajo productivo y reproductivo. Los trabajos en la producción agrícola familiar o la costura para afuera resultan así, por ejemplo, estrategias desarrolladas por las mujeres para poder atender al mismo tiempo sus responsabilidades domésticas y extradomésticas.

Por otra parte, esta clase social es un poco crítica de la poca comunicación de sus progenitores, es decir, de la inexistencia de diálogo y explicaciones que resultan fundamentales para la vida. La falta de educación sexual, la relevancia de la educación en general, etc., son así transmisiones que dicen no haber recibido pero que procuran legar a sus descendientes.

Y después... en el sentido de guiarles, hablarles, cosa que nuestros padres no lo hacían. Que te explicaran... O que vos hoy de grande te das cuenta que no te contaron. De los peligros que puede tener una mujer en la calle. De qué hay que cuidarse. De cómo hay que cuidarse para, ponele, que si salís con un chico cómo hay que cuidarse (Entrevista $\mathrm{n}^{\circ} 16$, reproducción de la clase trabajadora).

En las trayectorias de la clase trabajadora, la trasmisión se vincula con algunos saberes manuales, de oficio, y con oportunidades laborales para la inserción en el mercado del trabajo precario o informal. Ahora bien, esa transmisión de oportunidades en esta clase social adquiere cierta fluctuación, pues, por un lado, los padres y madres transmiten de manera consciente una posibilidad que orienta a sus hijos a vivir las mismas condiciones materiales limitadas que ellos, y, por otro lado, se ocupan de trasmitir el deseo de que sigan caminos laborales totalmente diferentes para alcanzar un mejor trabajo y posición social, aunque ello implique una transmisión que va en contra de la herencia posible o de su tradición familiar.

\section{Reflexiones finales}

En este artículo nos preguntamos por el lugar que ocupa la transmisión familiar en la configuración de las trayectorias de clase. En particular enfocamos la mirada en las trayectorias de reproducción y ascenso social de las distintas clases sociales y analizamos la importancia relativa y diferencial que la herencia va adquiriendo en los caminos laborales construidos y que aporta elementos para comprender cómo se construyen las desigualdades sociales.

Observamos así que, mientras en las trayectorias de la clase de servicio las familias suelen transmitir un conjunto de oportunidades formativas, materiales y laborales a sus herederos que posibilitan la inserción y desarrollo de trayectorias laborales en la misma clase social, en las trayectorias hacia la clase de servicios, la transmisión familiar es más simbólica que material, pues, si bien las oportunidades laborales y materiales legadas constituyen soportes disponibles, los mismos pierden importancia frente a la relevancia que adquiere la transmisión de deseos, vocaciones, aspiraciones, proyecciones y moralidades familiares que promueven el ascenso social y que, al ser especialmente valoradas y apropiadas 
por los descendientes, propician decisiones y acciones orientadas a alcanzar una mejor posición en la estructura social.

En las trayectorias de la clase intermedia se advierte, por su parte, que la transmisión se centra en oportunidades educativas y laborales que son apropiadas por las nuevas generaciones y son puestas en juego en el despliegue de sus caminos ocupacionales en la misma clase social de origen.

Las trayectorias hacia la clase intermedia muestran, en cambio, que la transmisión familiar adquiere ribetes particulares, pues una parte del legado es aceptado y apropiado por los descendientes, y otra parte es criticado y resignificado. Así, mientras la herencia aceptada se vincula con las oportunidades de acceso y terminalidad educativa del nivel medio y con algunos saberes que pudieron ser trasmitidos, por otro lado, se produce cierto distanciamiento de la transmisión de los valores de la clase trabajadora, relacionada con el sacrificio en el trabajo y sus recompensas, que es cuestionada y resignificada en estas trayectorias de ascenso social.

Finalmente, en las trayectorias de la clase trabajadora el legado familiar se relaciona con saberes manuales y de oficio, y con una transmisión de oportunidades laborales precarias e informales que encaminan las trayectorias de los hijos y sobrinos hacia la reproducción de su posición de clase y el deseo frustrado de ir más allá de la herencia para alcanzar mejores condiciones de vida.

Así, los trabajadores de la clase de servicios desarrollan trayectorias signadas por la transmisión y acumulación de ventajas formativas, materiales y laborales, y por las instituciones a las que tienen acceso. Los trabajadores que logran alcanzar la clase de servicios despliegan recorridos signados más por la trasmisión simbólica que material, transmisión encarnada en deseos que se pueden alcanzar a partir de las apropiaciones y disposiciones subjetivas y de las institucionalidades a las que puede recurrir. Los trabajadores de la clase intermedia despliegan trayectorias con ventajas relativas relacionadas con las oportunidades educativas medias y laborales que les son legadas y los soportes institucionales a que tienen acceso. Los trabajadores que ascienden a la clase intermedia configuran trayectorias ambivalentes, de aceptación del legado formativo y rechazo y resignificación de los valores heredados, que se articulan con los limitados apoyos institucionales. Finalmente, las clases trabajadoras construyen trayectorias en las cuales la trasmisión heredada se expresa en una acumulación de desventajas y deseos que no pueden ser alcanzados a través del acceso y uso de instituciones de la sociedad en la que viven.

Mientras en las trayectorias de la clase de servicios, intermedia y trabajadora las herencias transmitidas tienen un papel importante en la reproducción de las clases, en las trayectorias hacia la clase de servicios e intermedia, la transmisión invita a la apropiación y el rechazo del legado para reorientar los recorridos esperados. De esta forma, la transmisión simbólica de deseos, aspiraciones, vocaciones, proyecciones y moralidades $-\mathrm{y}$ la apropiación subjetiva de dicha herencia en la clase de servicios con las distintas aristas de la transmisión en la clase intermedia- ocupan un lugar destacado en estas trayectorias en tanto se transforman en condiciones de posibilidad, en factores distintivos que, articulados con otros elementos relevantes, como los soportes familiares e institucionales y la situación contextual, permiten comprender los procesos de ascenso que rompen la reproducción social. 
Las trasmisiones familiares (materiales, formativas, laborales, pero también simbólicas) y el lugar que ocupan en la construcción de las trayectorias pueden ser pensados, en suma, como elementos que enriquecen el estudio de las desigualdades sociales, en tanto posibilitan comprender cómo se alcanzan distintas posiciones en la estructura social de una manera dinámica, es decir, cómo la trasmisión familiar se encadena en la temporalidad con los contextos y las oportunidades institucionales y contextuales presentes en la sociedad, lo que da lugar a la configuración de trayectorias de clase previsibles e imprevisibles, que pueden reproducir o reconfigurar los destinos sociales esperables.

\section{Referencias}

Assusa, G. (2019). Repertorios de legitimación e impugnación moral de las desigualdades. Un estudio de las fronteras simbólicas de clase en Gran Córdoba, Argentina (2003-2015). Revista mexicana de ciencias politicas y sociales, 64 (237), 315-340. https://doi.org/10.22201/fcpys.2448492xe.2019.237.67329

Benza, G. (2014) El estudio de las clases medias desde una perspectiva centrada en las desigualdades en oportunidades de vida. Cuadernos de investigación en desarrollo. México: Programa Universitario de Estudios del Desarrollo. Universidad Nacional Autónoma de México.

Bertaux, D y Bertaux Wiane, I. (1997) Heritage and its linaje. A case history of transmission and social mobility over five generation. En Bertaux D. y Thompson P. Pathways to social class. (pp. 62 - 97) New York. Oxford University press.

Bertaux, D. y Thompson, P. (1997) Introduction. En Bertaux D. y Thompson P. Pathways to social class (PP. 27-56) New York. Oxford University press.

Bourdieu, P. (1997). Razones prácticas. Barcelona: Anagrama.

Bourdieu, P. (2011) La distinción. Criterios y bases sociales del gusto. Buenos Aires: Taurus.

Chávez Molina, E.; Pla, J.; Molina Derteano, P. (2011) Entre la adscripción, la estructura y el logro: determinantes de la movilidad social. Ministro Rivadavia, sur del Gran Buenos Aires, 2008- 2009. Revista Lavboratorio (24), 104-119.

Dalle, P. (2010) Cambios en el régimen de movilidad social intergeneracional en el Área Metropolitana de Buenos Aires (1960-2005). Revista Latinoamericana de Población, 4 (7),149-173. https://doi.org/10.31406/relap2010.v4.i2.n7.5

Dalle, P. (2013) Movilidad social ascendente de familias migrantes de origen de clase popular en el Gran Buenos Aires. El entramado de condiciones, recursos y experiencias que favorece el ascenso social. Trabajo y Sociedad, (21) 373 - 401.

Dubet, F. (2011) Repensar la Justicia Social. Buenos Aires: Siglo XXI.

Eguía, A. y Ortale, S. (2007) Los significados de la pobreza. Buenos Aires: Biblos.

Erikson, R y Goldthorpe, J. (1993) The Constant Flux: A Study of Class Mobility in Industrial Societies. Oxford: Oxford University Press.

Goffman, E. (1981). La presentación de la persona en la vida cotidiana. Buenos Aires: Amorrortu.

Goldthorpe, J. (1987) Social mobility and class structure in modern Britain. Oxford: Clarendon Press.

Gutiérrez, A. (2004) Pobre' como siempre. Estrategias de reproducción de la pobreza. Códoba: EDUVIM. 
Gutiérrez, A. y Mansilla, H. (2015) "Clases y reproducción social: el espacio social cordobés en la última década" Política y Sociedad Madrid, 52,409 - 444.

Jiménez Zunino, C. y Assusa, G. (2017) ¿Desigualdades de corta distancia? Trayectorias y clases sociales en Gran Córdoba, Argentina. Revista Mexicana de Sociología, 79(4), 837-87.

Jiménez, C. (2019) Modo de reproducción escolar en las clases sociales cordobesas. Un análisis desde las transmisiones intergeneracionales. Revista Temas Sociológicos (25). Santiago de Chile p. $291-327$

Jorrat, R. (1997). En la huella de los padres: Movilidad ocupacional en el Buenos Aires de 1980. Desarrollo Económico (37), 91-116.

Jorrat, J. R. (2000). Estratificación social y movilidad. Un estudio del área metropolitana de Buenos Aires. Tucumán: EUDET.

Kessler, G. y Di Virgilio, M. (2005) La nueva pobreza urbana: dinámica global, regional $y$ argentina en las últimas dos décadas. Santiago de Chile: CEPAL.

Kessler, G. y Espinoza, V. (2003) Movilidad social y trayectorias ocupacionales en Argentina: rupturas y algunas paradojas en el caso de Buenos Aires. Serie Políticas Sociales (66), Santiago de Chile: CEPAL

Lahire, B. (2002) Portraits Sociologiques. Paris: Nathan.

Minujin, A. y Kessler, G. (1995), La Nueva pobreza en la Argentina. Buenos Aires: Temas de Hoy-Planeta.

Muñiz Terra, L. (2012a) Carreras y trayectorias laborales: una revisión crítica de las principales aproximaciones teórico-metodológicas para su abordaje. Revista Latinoamericana de Metodología de las Ciencias Sociales, 2 (1), 36-55.

Muñiz Terra, L. (2012b) Los (ex) Trabajadores de YPF. Trayectorias laborales a veinte años de la privatización. Buenos Aires: Espacio Editorial.

Muñiz Terra, L. (2018). Biographical events and milestones: a methodological proposal to analyze narratives of life. Forum: Qualitative Social Research, 19 (2), 1-25.

Muñiz Terra, L.; Iucci, M. y Pla, J. (2019) Aproximaciones a la estructura social del Gran La Plata 2009 - 2016. Las desigualdades sociales entre el kirchnerismo y Cambiemos. V Seminario Internacional Desigualdad y Movilidad Social en América Latina. Santiago de Chile, Chile.

Patton, M. (2002) Qualitative Evaluation and Research Methods. Thousand Oaks, California: Sage.

Plá, J y Chávez Molina, E. (2011). La desigualdad dinámica: una indagación sobre las probabilidades y los canales de ascenso social en una localidad periférica del Gran Buenos Aires. Revista de Estudios Maritimos y Sociales, (4), 137-147.

$\mathrm{Pla}$, J. (2016) Condiciones objetivas y esperanzas subjetivas. Movilidad social y marcos de certidumbre. Un abordaje multidimensional de las trayectorias de clase. Región Metropolitana de Buenos Aires durante los dos mil. Buenos Aires: Autores de Argentina.

Reygadas, L. (2004) Las redes de la desigualdad: un enfoque multidimensional. Politica y cultura, otoño (22) 7-25. Xochimilco, México: UAM

Salvia, A. (2012). La trampa neoliberal. Un estudio sobre los cambios en la heterogeneidad estructural y la distribución del ingreso en la Argentina: 1990-2003. Buenos Aires: EUDEBA.

Salvia, A. y Chávez Molina, E. (2007) Sombras de una marginalidad fragmentada. Aproximaciones a la metamorfosis de los sectores populares de la Argentina. Buenos Aires: Miño y Dávila. 
Saraví, G. (2015). Juventudesfragmentadas: socialización, clase y cultura en la construcción de la desigualdad. México: FLACSO-México.

Savage, M. (2014). A new model of social class? Findings from BBC's great British class survey experiment. Sociology, 47 (2), Reino Unido.

Sen, A. (1999). Nuevo examen de la desigualdad. Madrid: Alianza Editorial.

Teriggi, F. (2015) De la trayectoria en singular a las trayectorias en plural, en F. Teriggi y J. Lopez (coord.) Directores que hacen Escuela. Buenos Aires: OEI.

Therborn, G. (2011). Inequalities and Latin America from the Enlightenment to the 21st Century. En Working paper Series $N^{\circ} 1$. Berlin: Desigualdades.net. Research Network on Interdependent Inequalities in Latin America Recuperado de: http://www.desigualdades.net/Working_Papers/Search-Worki ng-Papers/Working-Paper-1__Inequalities-and-Latin-America_/index.html

Thompson, P. (1997). Women, man and transgenerational family influences in social mobility. En Bertaux, D. y Thompson, P. Pathways to social class. (p. 32-61). New York. Oxford University Press.

Tilly, C. (2000). La desigualdad persistente. Buenos Aires: Manantial.

Varesi, G. (2009) La configuración del modelo postconvertibilidad: Políticas y clases. Algunas claves para su caracterización, 2002-2007. Cuestiones de Sociología 5-6, $27-54$.

Wilkinson, R. y Pickett, K. (2009): The Spirit Level. London: Allen Lane.

Znaniecki, F. (1934) The Method of Sociology. New York: Farrar \& Rinehart.

\section{Notas}

1 Este escrito se enmarca en el proyecto "Trayectorias laborales, generaciones y clases sociales: un análisis de las desigualdades sociales en el Gran la Plata”, (2003-2019), 11/ H859 y PICT 2015-0928, dirigido por Leticia Muñiz Terra y financiado por Agencia I + D + I y Ministerio de Educación de la Nación. Part of this article was also elaborated in the context of INCASI Network, an European project that has received funding from the European Union's Horizon 2020 research and innovation programme under the Marie Skłodowska-Curie GA No 691004 and coordinated by Dr. Pedro LópezRoldán. This article reflects only the author's view and the Agency is not responsible for any use that may be made of the information it contains.

2 La autora del texto reconoce y adhiere a la deconstrucción de las miradas patriarcalistas vinculadas a subsumir en el género masculino al género femenino. Sin embargo, en este texto, solo por una cuestión de simplificación, se utilizará los géneros de manera indistinta: sobre todo cuando se adhiera a tío/tía, abuelo/abuela, etc. 